\title{
Eco-driving simulation: evaluation of eco-driving within a network using traffic simulation
}

\author{
I. Kobayashi ${ }^{1}$, Y. Tsubota ${ }^{2}$ \& H. Kawashima ${ }^{1}$ \\ ${ }^{1}$ School of Science for Open and Environmental Systems, \\ Keio University, Japan \\ ${ }^{2}$ Institute for Natural Sciences, J. F. Oberlin University, Japan
}

\begin{abstract}
Curbing Global Warming necessitates the reduction of greenhouse gases. Motor-vehicle companies are attempting to improve engine efficiencies but are constrained by technical limitations. Hence we try to change driving behaviour to reduce fuel consumption. A change in driving style/habit to promote fuel efficiency and hence to reduce $\mathrm{CO}_{2}$ emissions is known as 'eco-driving'. One such eco-driving style is to reduce sudden acceleration and deceleration. A report based on eco-driving shows a $10-20 \%$ reduction in fuel consumption per vehicle; and hence, eco-driving is promoted by Japan's Ministry of Environment. However, a mass of eco-drivers might impede traffic flow which fosters traffic congestion. This study evaluates eco-driving with respect to a traffic network versus an individual vehicle. We performed field measurements of a vehicle's speed and acceleration versus normal and eco-driving styles. We input the data into a micro-traffic simulator (VISSIM, PTV AG). We built a simple road network with two signalized intersections. Ten simulations were done using different random-number seeds. We found that eco-driving increases environment load; i.e., carbon dioxide emission within the network during heavy-traffic volume. We propose an eco-driving control system and show its effectiveness.
\end{abstract}

Keywords: eco-driving, ITS, emission, $\mathrm{CO}_{2}$, traffic simulation. 


\section{Introduction}

Curbing Global Warming necessitates the reduction of greenhouse gases. The Kyoto Protocol of the United Nations Framework Convention on Climate Change was adopted in 1997. This protocol is an amendment to the international treaty on climate change, assigning mandatory emission limitations for the reduction of greenhouse gas emissions to the signatory nations. In this framework, Japan promised to decrease the 1990-level Carbon Dioxide $\left(\mathrm{CO}_{2}\right)$ emissions by $6 \%$ until 2012. Unfortunately, Japan's total $\mathrm{CO}_{2}$ emission has increased by $8.2 \%$ so an adequate strategy must be found to reduce $\mathrm{CO}_{2}$ emissions.

Figure 1 shows $\mathrm{CO}_{2}$ emissions as reported by Japan's industrial sectors. Japan's Ministry of the Environment (MOE) is trying to reduce $\mathrm{CO}_{2}$ emissions caused by its second-highest producer, the transportation sector. Motor-vehicle companies are attempting to improve engine efficiencies but are constrained by technical limitations.

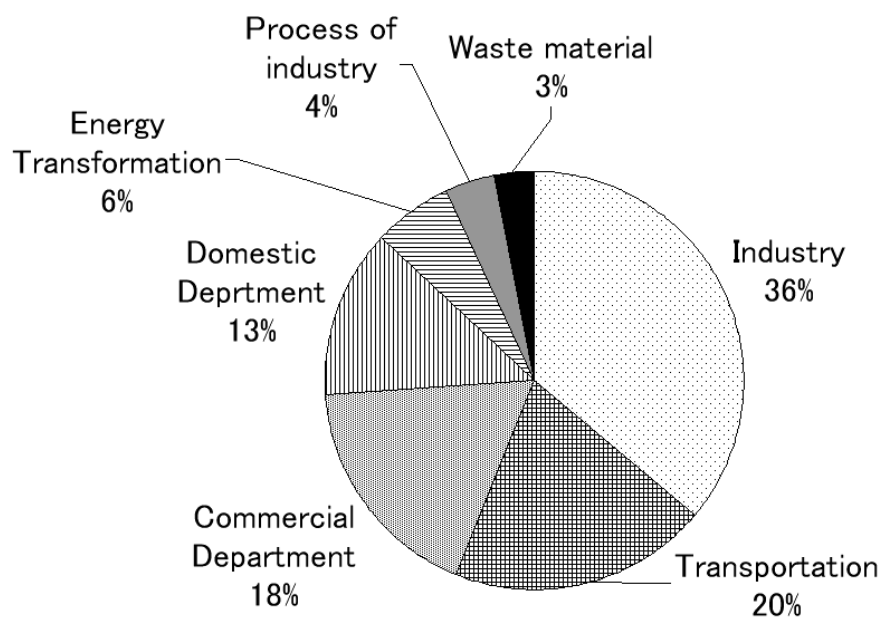

Figure 1: $\quad \mathrm{CO}_{2}$ emission inventory in Japan (2004).

Hence we try to change driving behaviour to reduce fuel consumption. A change in driving style/habit to promote fuel efficiency and hence to reduce $\mathrm{CO}_{2}$ emission, is known as 'eco-driving'. A report based on eco-driving shows a 10$20 \%$ reduction in fuel consumption per vehicle; and hence, eco-driving is promoted by Japan's MOE. One such eco-driving habit is to reduce sudden acceleration and deceleration.

But slow acceleration tends to causes congestion and hence wastes fuel. So we believe that eco-driving would be more efficient within free flow versus heavier-congested traffic. In this paper, we report the following two topics:

1) To estimate environmental impacts due to eco-driving; and

2) To propose the eco-driving control system using the Intelligent Transport Systems (ITS). 


\section{Eco-driving}

'Eco-Driving' refers to the kind of driving behaviour that minimize fuel consumption and hence tail-pipe emissions; so there is an economical and an environmental benefit. Eco-Driving means:

a) No unnecessary idling;

b) Avoid sudden, sharp acceleration; and

c) Use engine brakes efficiently.

The Japan Automobile Federation (JAF) reports that fuel consumption of 5second idling is equivalent to staring up a warm engine. Thus, it's economical to stop idling beyond 5 seconds.

The JAF claims that an engine's working speed that is greater than 2500 $\mathrm{rev} / \mathrm{sec}$ is wasteful, particularly when starting an engine; so sharp acceleration should be avoided.

You can reduce fuel consumption if you engine-break efficiently. An automatic transmission allows the driver to effectively break the car by removing the foot from the accelerator pedal. A manual transmission requires the driver to change to a lower gear to produce the same effect. These engine-break techniques reduce unnecessary acceleration and sudden breaking. Therefore, efficient usage of engine breaks results in reduction of fuel consumption.

Speed and acceleration pattern data are required to evaluate eco-driving. We used two different vehicles to determine these patterns: Volkswagen Golf Touran GLI and Mazda Eunos_800. We monitored vehicle speed and acceleration every second using Global Positioning System (GPS). Eighteen drivers with the average age of 22 years were tested within a standard deviation of 1.2 years. A datum was determined using 'normal' driving behaviours; then eco-driving behaviours that use modest acceleration and deceleration were followed.

This is termed 'eco-driving' acceleration. Results are shown in figure 2 to 5 . We can confirm that deceleration is constant at $-0.5 \mathrm{~m} / \mathrm{s}^{2}$ when the vehicle speed is between $40 \mathrm{~km} /$ hour and $60 \mathrm{~km} /$ hour, as shown in Figure 5. This is the result of using the engine as a break.

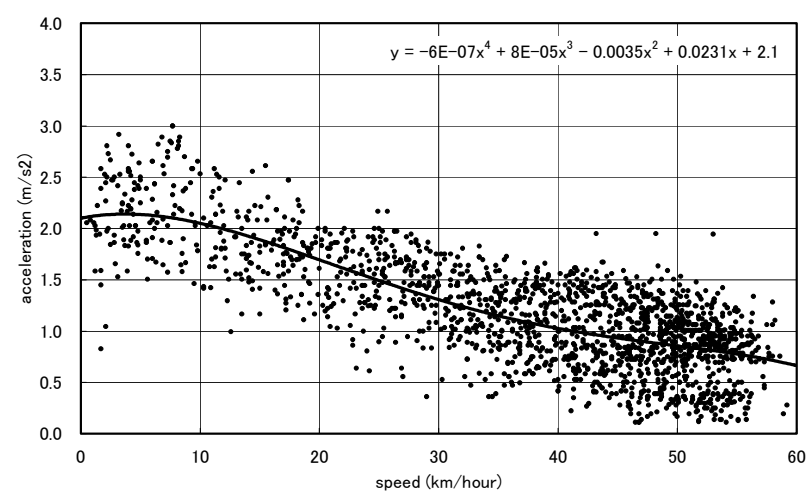

Figure 2: Normal-driving acceleration. 
744 Urban Transport XIII: Urban Transport and the Environment in the 21st Century

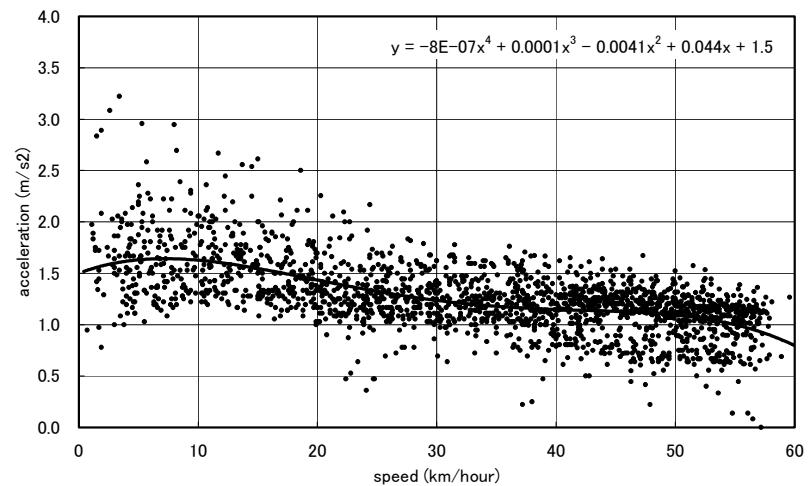

Figure 3: Eco-driving acceleration.

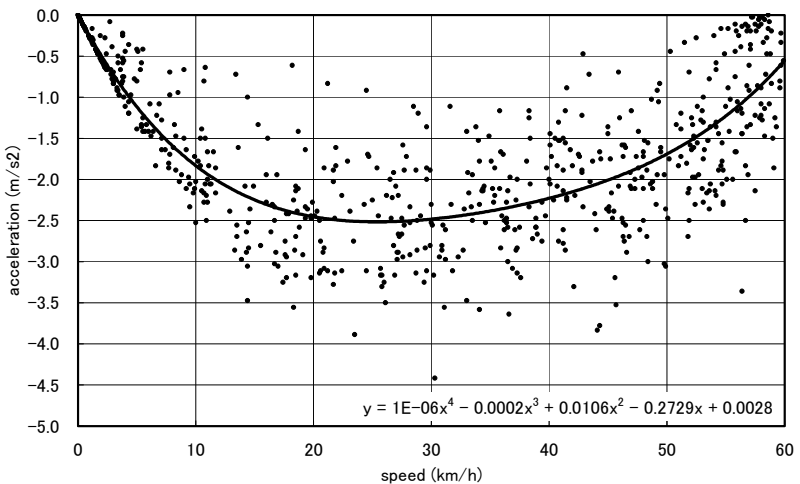

Figure 4: Normal-driving deceleration.

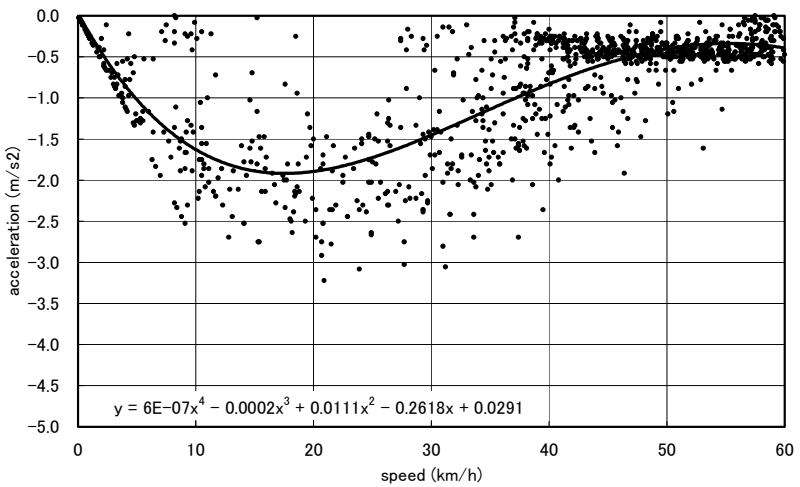

Figure 5: Eco-driving deceleration. 


\section{Traffic simulation of the eco-driving}

The effects of eco-driving were evaluated using the micro-traffic simulator 'Vissim' developed and distributed by PTV AG of Germany. Speed and acceleration pattern data obtained by field measurements are inputted into Vissim. Then, vehicle emissions for normal and eco-driving were computed from vehicle speed and acceleration.

A two-lane road network was used in the simulation. We used a $1000 \mathrm{~m}$ straight road with two traffic signals at $300 \mathrm{~m}$ and $600 \mathrm{~m}$, to simulate a one-hour run (Figure 6). These signals consist of 30 -second blue followed by 3 -second yellow and 27-second red light. The offset of consecutive signals was not considered for simplicity. The simulations were executed for different traffic volumes. They changed from 100 vehicles/hour to 1800 vehicles/hour. Ten simulations for each traffic volume were executed using ten different randomnumber seeds. Vehicle-emission changes were evaluated using the average of ten simulations. The improvement ratio, $\mathrm{R}$ is defined as following equation.

$$
\mathrm{R}=(\text { En - Ee }) / \text { En } \times 100
$$

where En and Ee are total vehicle emission of normal-driving and eco-driving, respectively.

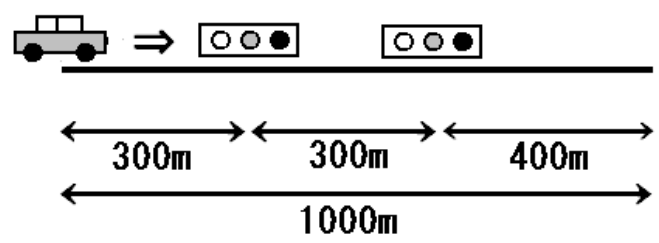

Figure 6: Road network in the traffic simulation.

Figure 7 shows the relationship between the improvement ratio for $\mathrm{CO}_{2}$ emission and traffic volume. The improvement ratio is about $+3 \%$ until 1700 vehicles/hour which means eco-driving is effective. However, the improvement ratio drops off severely when traffic volume exceeds 1700 vehicles/hour. This means the benefits of eco-driving wanes with heavy-traffic volume. This is because an eco-driving car accelerates so slowly that it retards following cars, and hence, causes traffic congestion that induce greater $\mathrm{CO}_{2}$ emission.

The travel time of eco-driving is similar to normal driving until traffic volume reaches 1700 vehicles/hour as shown in figure 8. Beyond that, eco-driving retards traffic flow, under the heavy traffic condition.

Results suggest that uncontrolled eco-driving can retard traffic flow and hence, induce greater $\mathrm{CO}_{2}$ emission, particularly in heavy traffic. Hence we propose an eco-driving control system that maximizes the eco-driving benefits per traffic network versus per individual vehicle. 


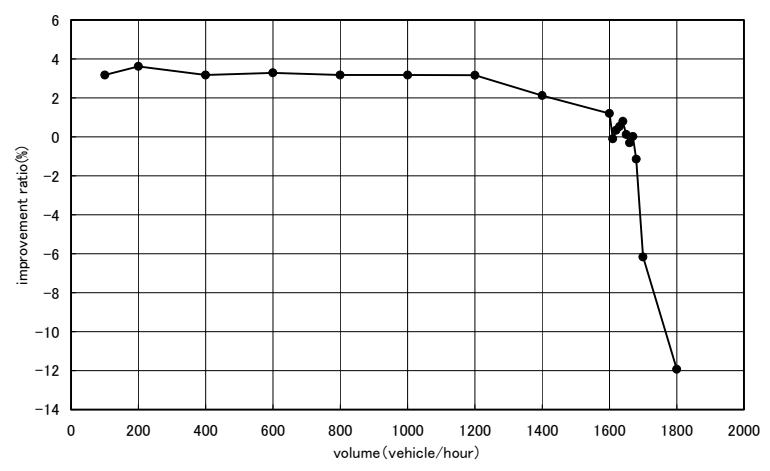

Figure 7: The improvement ratio of $\mathrm{CO}_{2}$ emission.

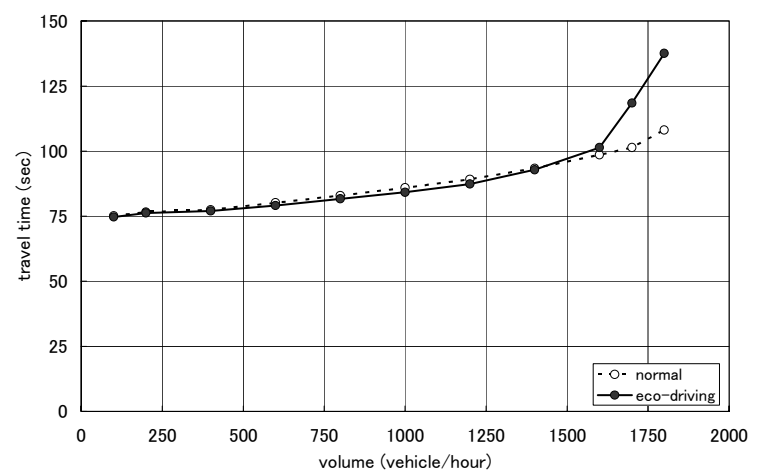

Figure 8: The comparison of travel times.

The algorithm of an eco-driving control system is shown in Figure 9. The average flow-speed $\mathrm{V}$ was monitored using a vehicle detector and compared with threshold speed $V_{1}$ per time interval $\left(T_{1}-\right.$ minutes $)$. In this study, we set $T_{1}$ to 20 minutes, and $\mathrm{V}_{1}$ to $45.2 \mathrm{~km} /$ hour. We ran traffic simulations to check the ecodriving control system. In these simulations, we change the road length to $10,000 \mathrm{~m}$ and signal spacing to $600 \mathrm{~m}$ as shown in figure 10 .

Figure 11 implies that control algorithm can be efficient when the heavy traffic volume although eco-driving drops off severely around 2700 vehicles/hour.

We can confirm that eco-driving tends to retard traffic with volumes greater than 2700 vehicles/hour, and the control algorithm solves disadvantages of ecodriving for heavy traffic as shown in Figure 12. 


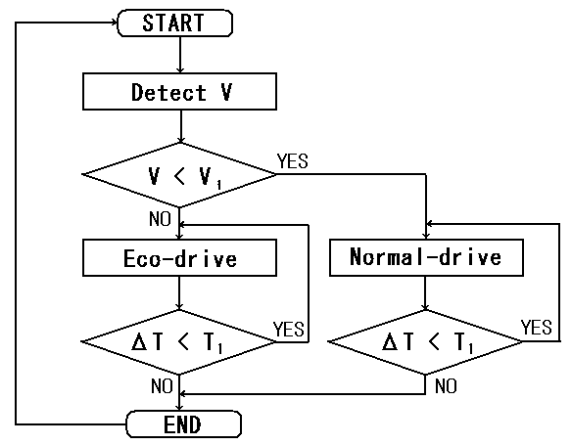

Figure 9: Eco-driving control algorithm.

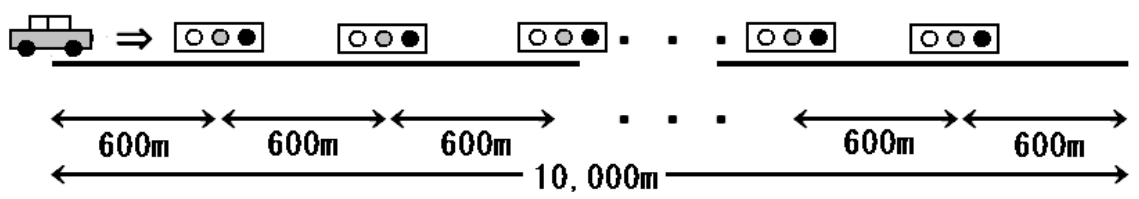

Figure 10: Road network for the eco-driving control system simulation.

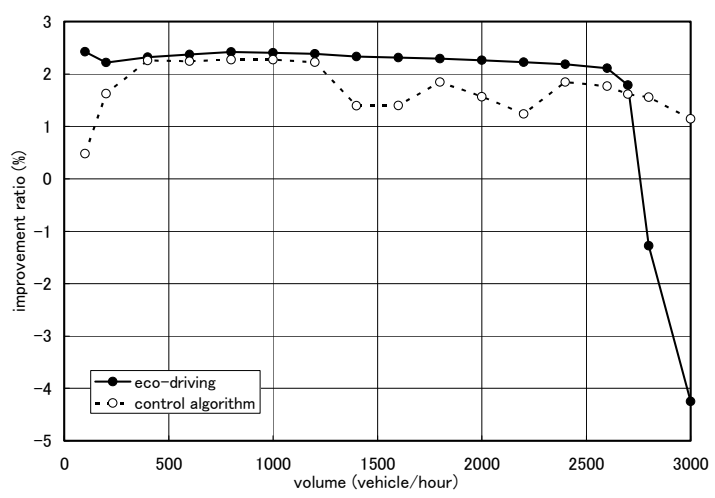

Figure 11: The improvement ratio of $\mathrm{CO}_{2}$ emission.

\section{Eco-driving simulation with real world data}

We have tested eco-driving using traffic simulation with real-world data of 19 April 2006 at Sin-Ishikawa intersection in Yokohama, Japan (Figure 13). We input traffic volume per hour, signal cycles, route decision, and the ratio of vehicle types (i.e., compact vehicle, heavy-goods vehicle, and motor cycle). We also input pedestrian data. Three different scenarios were simulated: a) normaldriving for all vehicles, b) eco-driving for all vehicles, and c) eco-driving for $50 \%$ of vehicles. 


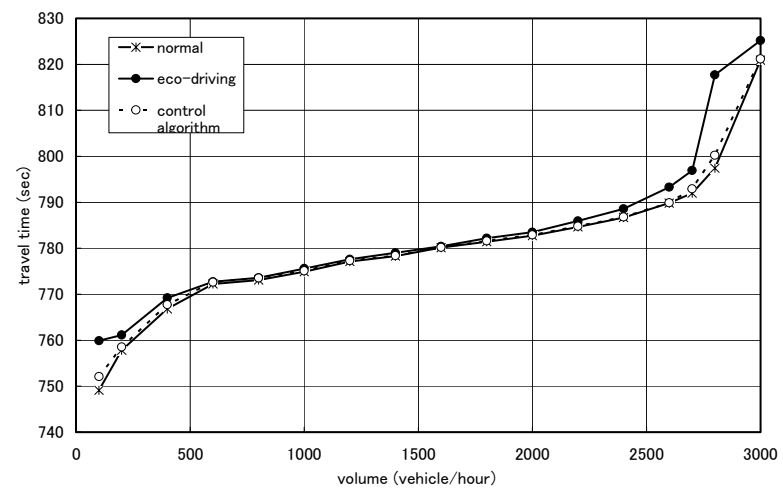

Figure 12: The comparison of travel times.

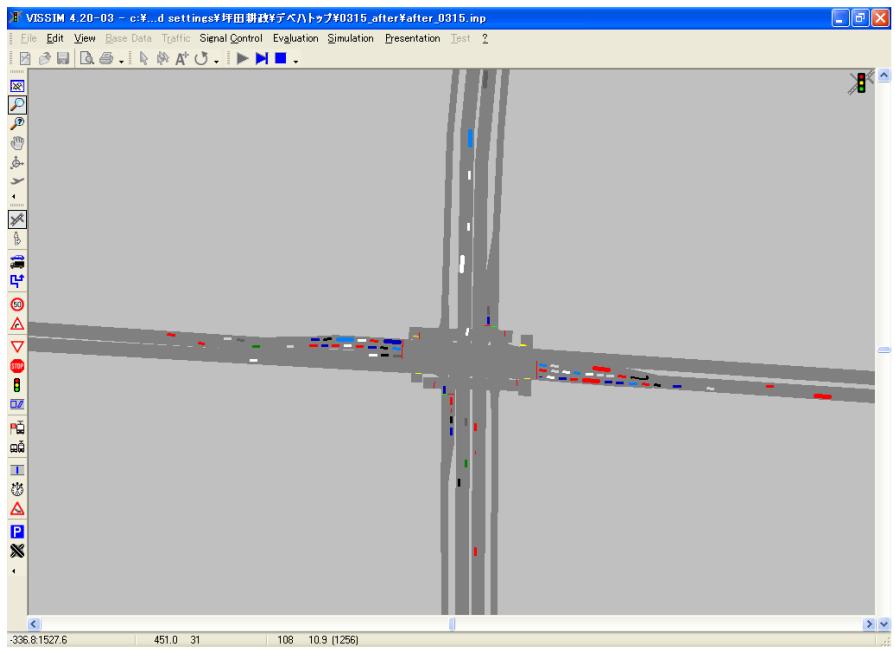

Figure 13: Shin-Ishikawa intersection.

Diurnal variations of $\mathrm{CO}_{2}$ and $\mathrm{NO}_{\mathrm{x}}$ emissions are shown in Figure 14 and 15, respectively. Traffic volume changes are also shown in these figures. There is only one morning peak for the heavy-good vehicle (HGV) but there are morning and evening peaks for the compact vehicle. Table 1 shows the comparison of total emissions per scenario. According to the Table. 1, eco-driving emits less $\mathrm{CO}_{2}$ than normal-driving. However, Figure 14 shows $\mathrm{CO}_{2}$ emission is greater for eco-driving than normal-driving around 7:00 a.m. due to the morning rushhour when eco-driving is less efficient. It is inferred that the effects of ecodriving depends not only on traffic volume but also on traffic composition. 


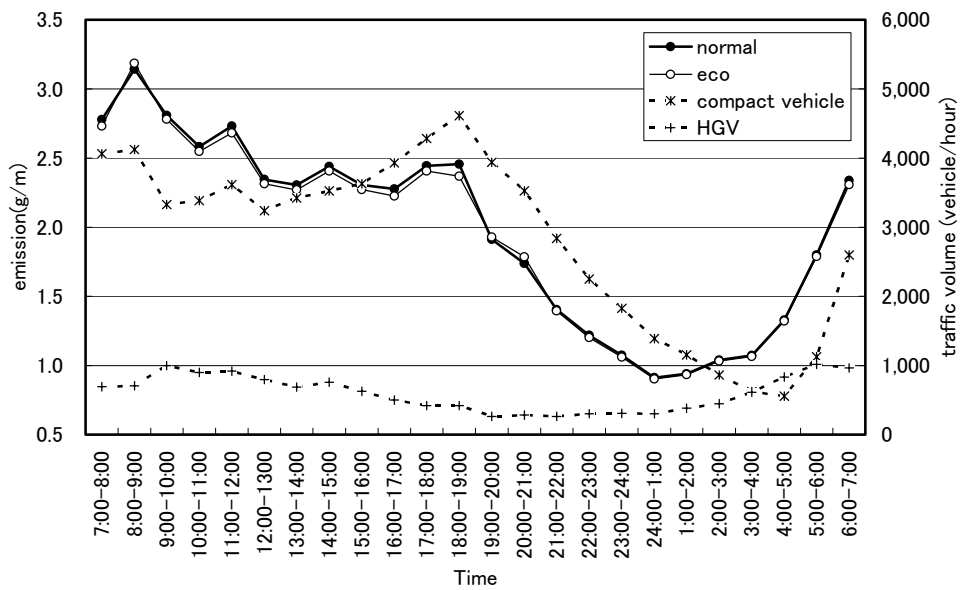

Figure 14: $\quad \mathrm{CO}_{2}$ emission in Shin-Ishikawa intersection.

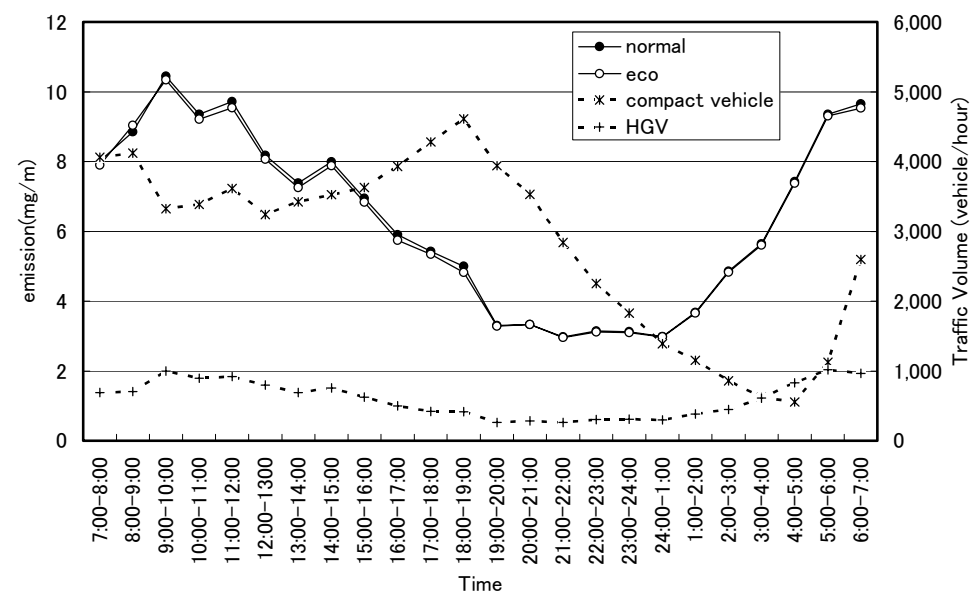

Figure 15: $\quad \mathrm{NO}_{\mathrm{x}}$ emission in Shin-Ishikawa intersection.

Table 1: $\quad$ Comparison of the total emission for each scenario.

\begin{tabular}{|l|c|c|c|c|}
\hline \multirow{2}{*}{} & \multicolumn{2}{|c|}{$\mathrm{CO}_{2}$ (g/hour) } & \multicolumn{2}{c|}{$\mathrm{NO}_{\mathrm{x}}$ (g/hour) } \\
\cline { 2 - 5 } & mean & $\mathrm{sd}$ & mean & $\mathrm{sd}$ \\
\hline a) normal & 4729.49 & 45.129 & 15.26 & 0.135 \\
\hline b) eco-driving & 4681.83 & 33.227 & 15.09 & 0.112 \\
\hline c) $50 \%$ eco-driving & 4680.26 & 32.862 & 15.09 & 0.103 \\
\hline
\end{tabular}




\section{Summary}

Generally speaking, eco-driving is economical and efficient for the environment. Most studies of eco-driving evaluate eco-driving as a unit car. In this study, we evaluate eco-driving with respect to a network versus an individual vehicle. As a result, we found that eco-driving increases environment load; i.e., carbon dioxide emission, within a network under heavy traffic. Also, we showed that an ecodriving control system can improve eco-driving efficiency within a traffic network. We showed that an eco-driving system can easily be implemented using intelligent transport systems such as a variable-message board. However, we showed the necessity to investigate an eco-driving control system using traffic simulations with real-world data in order to reduce $\mathrm{CO}_{2}$ emissions from the road network.

\section{References}

[1] Oneyama, H, Oguchi, T., Kuwahara, M., Estimation model of vehicle emission considering variation of running speed, Journal of the Eastern Asia Society for Transportation Studies, 4(5), pp.105-117, 2001.

[2] PTV, VISSIM4.10 USER MANUAL, PTV, Karlsruhe, Germany, 2005.

[3] Foundation for promoting personal mobility and ecological transportation, http://www.ecomo.or.jp/top_ecodrive/index.htm 